Service social

\title{
Les déterminants sociaux de la santé et la violence conjugale : Quels sont les liens?
}

\section{Luc Thériault et Carmen Gill}

Volume 53, numéro 1, 2007

URI : https://id.erudit.org/iderudit/017989ar

DOI : https://doi.org/10.7202/017989ar

Aller au sommaire du numéro

Éditeur(s)

École de service social de l'Université Laval

ISSN

1708-1734 (numérique)

Découvrir la revue

Citer cet article

Thériault, L. \& Gill, C. (2007). Les déterminants sociaux de la santé et la violence conjugale : Quels sont les liens ? Service social, 53(1), 75-89.

https://doi.org/10.7202/017989ar
Résumé de l'article

Dans cet article, nous discutons comment conceptualiser les « déterminants sociaux de la santé » parmi les causes de la violence conjugale. Dans la première partie du texte, nous présentons brièvement les déterminants sociaux de la santé et une partie de ce que nous en savons. Aux deuxième et troisième parties, nous parlons de la violence faite aux femmes (signifiant ici surtout la violence physique ou l'abus sexuel de la part d'un conjoint) et comment elle peut être liée aux conditions de vie des femmes en ce qui a trait aux déterminants sociaux de la santé. En conclusion, nous soulignons que l'étude de la violence faite aux femmes dans la perspective des déterminants sociaux de la santé nous mène à comprendre que diverses mesures en matière de politiques sociales (par exemple dans les domaines du logement, du soutien au revenu et des services de garde) pourraient aider à réduire ce type de violence et à améliorer la santé des populations. 


\title{
Les déterminants sociaux de la santé et la violence conjugale : Quels sont les liens?
}

\author{
LUC THÉRIAULT \\ Professeur agrégé \\ Département de Sociologie \\ Université du Nouveau Brunswick \\ Carmen GILL \\ Directrice \\ Le Centre Muriel McQueen Fergusson \\ pour la recherche sur la violence familiale \\ Fredericton, Nouveau Brunswick
}

Dans cet article, nous discutons comment conceptualiser les « déterminants sociaux de la santé » parmi les causes de la violence conjugale. Dans la première partie du texte, nous présentons brièvement les déterminants sociaux de la santé et une partie de ce que nous en savons. Aux deuxième et troisième parties, nous parlons de la violence faite aux femmes (signifiant ici surtout la violence physique ou l'abus sexuel de la part d'un conjoint) et comment elle peut être liée aux conditions de vie des femmes en ce qui a trait aux déterminants sociaux de la santé. En conclusion, nous soulignons que l'étude de la violence faite aux femmes dans la perspective des déterminants sociaux de la santé nous mène à comprendre que diverses mesures en matière de politiques sociales (par exemple dans les domaines du logement, du soutien au revenu et des services de garde) pourraient aider à réduire ce type de violence et à améliorer la santé des populations.

Mots clés : santé, femmes, violence conjugale, politique sociale.

Social determinants of health have a considerable impact on the health status of individuals. It is also known that being abuse by an intimate partner can cause serious and long lasting negative effects to women's health. In this paper, we discuss the under-studied possible links between certain determinants of health and woman abuse. While we postulate that in some cases this form of criminal victimization can be an intervening factor between these determinants and health outcomes, we also insist on the complexity inherent to these phenomena. In conclusion, we stress that realizing that links probably exist between social determinants of health and woman abuse should make us more attentive to the potential of various social policy initiatives (in areas such as housing, childcare, and income support or community development) for both improving health, and reducing women's victimization by intimate partners.

Keywords: health, women, domestic violence, social policy. 


\section{INTRODUCTION}

Des études récentes (Wuest et Merritt-Gary, 2004) soulignent qu'il est de plus en plus admis que la violence faite aux femmes a des répercussions graves et souvent à long terme, et des effets qui sont onéreux tant pour les personnes que pour le réseau de soins de santé ${ }^{1}$. Ce type de recherche se rapporte à ce qui arrive à la santé et au bienêtre des femmes à la suite d'une violence perpétrée par un conjoint. Par contre, les enjeux dont nous discuterons dans ce texte se rapportent à ce qui se produit avant l'incident violent : comment conceptualiser des facteurs qu'on appelle les « déterminants sociaux de la santé » parmi les causes de la violence dans le couple. Les enjeux sont certes extrêmement complexes, et nous ne prétendons pas démêler ici tous les liens ou les relations causales possibles entre les déterminants de la santé et la violence conjugale. Notre objectif est beaucoup plus modeste : alimenter une discussion de ces enjeux dans l'espoir que ce soit utile à long terme pour améliorer la santé et rendre les collectivités locales plus sûres et plus saines, surtout en ce qui a trait aux femmes et aux enfants.

Dans la première partie de ce document, nous présentons brièvement les déterminants sociaux de la santé et une partie de ce que nous en savons. Aux deuxième et troisième parties, nous parlons de la violence faite aux femmes (signifiant ici surtout la violence physique ou l'abus sexuel de la part d'un conjoint) et analysons comment elle peut « résulter » en partie de la condition des femmes en ce qui a trait aux déterminants sociaux de la santé. Évidemment, cet exposé n'est qu'une simplification très approximative de la réalité. En conclusion, nous soulignons que l'étude de la violence faite aux femmes à l'aide de la perspective des déterminants sociaux de la santé nous mène à comprendre que diverses mesures en matière de politiques sociales (par exemple dans les domaines du logement, du soutien au revenu et des services de garde) pourraient aider à réduire la violence et à améliorer la santé.

\section{DÉTERMINANTS SOCIAUX DE LA SANTÉ}

Depuis un bon moment, une grande part de ce que nous entendons ou lisons au sujet de la santé est axée sur l'apport de la médecine et du réseau des soins de santé. D'une certaine manière, les médias voient encore les choses sous l'angle de la « crise de la santé » qui ne cesse de se reproduire. Par contre, depuis la publication du Rapport Lalonde en 1974, nous savons que le réseau de la santé et la santé des populations sont deux choses différentes, et qu'investir des ressources toujours plus importantes dans le réseau de la santé n'a pas nécessairement pour résultat une amélioration remarquable de la santé des populations, car la santé est influencée par d'autres facteurs (ou « déterminants ») qui se trouvent à l'extérieur du réseau des soins de santé. Nous savons donc que l'augmentation du nombre de lits dans les hôpitaux d'une province n'est pas une panacée pour améliorer la santé d'une population.

1. Au Canada, Greaves, Hankivsky et Kingston-Riechers (1995) ont estimé que le coût annuel de la violence entre conjoints dépassait les $408 \mathrm{M} \$$. 
En général, on estime que le réseau de la santé compte pour environ $25 \%$ de l'état de santé d'une population. On croit d'autre part que les facteurs biologiques et le bagage génétique comptent pour environ $15 \%$, le milieu physique pour $10 \%$, alors que les facteurs sociaux et économiques pour $50 \%$ de l'état de santé d'une population. Ainsi, des facteurs tels que l'éducation, l'emploi, le revenu, ainsi que les milieux sociaux et physiques où nous vivons, ont une influence sur notre santé à un niveau au moins aussi important - sinon plus - que la qualité et la disponibilité des services de santé.

TABLEAU 1

CE QUI DÉTERMINE L'ÉTAT DE SANTÉ

1. Revenu et statut social

2. Réseaux de soutien social

3. Éducation et alphabétisation

4. Emploi et conditions de travail

5. Milieu social (normes et valeurs)

6. Milieu physique (aliments, logement, habillement et loisirs)
7. Hygiène personnelle et habiletés d'adaptation

8. Sain développement des enfants

9. Biologie et bagage génétique

10. Accès à des services de santé de qualité

11. Sexe

12. Culture

Source : Santé Canada, Agence de santé publique du Canada (2005).

Qu'est-ce que ça signifie ? En clair, dire que le revenu et le statut social sont des déterminants de la santé, c'est dire que les gens qui ont un revenu plus élevé jouissent en général d'une meilleure santé et vivent plus longtemps que les gens à revenu moins élevé. II semble que cela ne soit pas uniquement lié à la capacité de s'assurer un logement sûr et d'acheter assez d'aliments de très bonne qualité, mais également au fait que les gens à revenu plus élevé jouissent aussi d'un plus grand contrôle sur leurs conditions de vie. Des chercheurs britanniques constatent dernièrement que les hommes qui ont un emploi de bas niveau (avec peu de contrôle sur leurs tâches quotidiennes) ont effectivement un rythme cardiaque plus élevé, ce qui pourrait expliquer pourquoi les hommes qui occupent des emplois peu rémunérés risquent davantage la cardiopathie - une tendance qu'on connaît depuis trente ans. Signalons qu'il y a entre le statut économique et social plus élevé et une meilleure santé un lien solide et bien établi du point de vue scientifique, quoique les mécanismes biologiques qui l'expliquent soient encore mal connus. De plus, on devrait se préoccuper non seulement du lien entre le statut socio-économique et l'état de santé, mais aussi du fait que l'ampleur de l'inégalité elle-même (l'écart entre les riches et les pauvres) est liée à la santé globale des sociétés. Plus grand est l'écart entre les niveaux de revenus observé, plus grands sont les problèmes sociaux et moins bon est l'état de santé de la population dans son ensemble.

Dire que les réseaux de soutien social sont des déterminants de la santé, c'est dire que les gens sont en meilleure santé quand ils se sentent en sécurité, soutenus par les autres et en lien avec eux - quand ils peuvent faire confiance aux membres de leurs 
familles et compter sur des amis, des voisins et des membres de la communauté en général. On croit que le soutien social est important, car il s'agit d'un facteur clé pour aider les individus à résoudre des problèmes et à survivre à des crises dans leur vie. Connaître quelqu'un à qui on peut se confier, quelqu'un sur qui on peut compter en temps de crise, quelqu'un à qui demander conseil, est bon pour la santé. Des études ont effectivement associé diverses conséquences pour la santé - depuis le risque d'angine de poitrine aux taux de décès prématuré - à la quantité de contacts sociaux et de soutien émotionnel. Le soutien social est particulièrement important au moment de la grossesse et il a été démontré qu'il avait une influence sur le poids du bébé à la naissance et sur son état général de santé. Des relations stables, significatives et importantes avec des amis, la parenté et des voisins, sont aussi très précieuses lorsqu'on élève des enfants. Les jeunes familles (et surtout les femmes) qui ne disposent pas de ce soutien, restent vulnérables face aux conséquences des tensions de la vie. C'est pourquoi les chercheurs disent que les relations sociales agissent comme « tampon » en période de tensions.

Dire que l'éducation est un déterminant de la santé, c'est dire que l'état de santé s'améliore selon le niveau d'éducation (qui est intimement lié au revenu et au statut social). L'éducation apporte des compétences utiles pour résoudre les problèmes et renforce l'impression de contrôle et le sentiment de maîtrise de sa propre situation dans la vie. Elle permet de meilleures occasions d'emploi, une plus grande sécurité du revenu et une plus grande satisfaction au travail. Elle accroît aussi la capacité à comprendre comment se garder en santé et à agir en conséquence, en choisissant d'éviter certains comportements (comme le tabagisme) et d'en adopter d'autres (par exemple l'activité physique) ${ }^{2}$.

Dire que les conditions d'emploi et de travail sont des déterminants de la santé, c'est dire qu'avoir un emploi exerce en général un effet positif sur la santé, alors que le chômage a un effet néfaste important, tant sur la santé physique que mentale. Cela signifie que les travailleurs ont tendance à être en meilleure santé, quand ils croient que leur emploi est assuré, que le travail qu'ils effectuent est important et valorisé, et lorsque leur milieu de travail offre aussi des possibilités de prendre des décisions et de s'épanouir du point de vue personnel.

Parler du genre comme déterminant de la santé, c'est reconnaître que les femmes et les hommes ont souvent différents rôles et responsabilités déterminés par la société, ainsi que des réalités sociales différentes. Les hommes et les femmes n'ont pas les mêmes occasions d'accéder à des ressources et de les contrôler, et les femmes gagnent encore en moyenne des revenus moindres que les hommes. Par conséquent, des disparités perdurent entre l'état de santé des hommes et celui des femmes, en

2. Comme l'indiquait un lecteur du texte, il ne s'agit pas uniquement de connaissance, mais aussi des ressources pour mettre cette connaissance à l'œuvre - il faut donc de l'argent ou des assurancesmaladie complémentaires pour réaliser plusieurs stratégies pour arrêter de fumer, et il faudra peut-être aussi de l'argent et d'autres ressources pour trouver le temps, les services de garde et les installations pour faire de l'exercice. 
raison des différences touchant les types de besoins et l'accès aux soins de santé, les comportements pour se garder en santé et les conditions socio-économiques.

Bien que les femmes vivent plus longtemps que les hommes, elles sont plus exposées à la dépression, à un stress excessif (souvent dû aux efforts pour équilibrer les vies professionnelle et familiale), à des maladies chroniques comme l'arthrite et les allergies, à des blessures et au décès imputables à la violence familiale (Comité consultatif fédéral-provincial-territorial sur la santé de la population, 1999 : viii).

Dire que la culture (c'est-à-dire la langue, l'identité ethnique ou raciale, la religion) est un déterminant de la santé, c'est dire que les valeurs acquises par l'entremise de votre socialisation en ce qui a trait à la santé et à la maladie auront vraisemblablement une influence sur les décisions que vous prendrez en ayant recours (ou non) aux services de santé, et sur le type de services auxquels vous ferez appel, etc.

Globalement, le fait de disposer d'un environnement sain, d'un revenu adéquat, d'un rôle significatif dans la société, d'un logement sécuritaire, d'un niveau de scolarité plus élevé et d'un certain soutien social dans la communauté, est lié à une meilleure santé et au mieux-être. Les déterminants sociaux de la santé sont donc des facteurs du milieu social qui renforcent la santé de la collectivité et de ses membres, ou qui y nuisent. Le savoir est une chose, mais le fait de prendre vraiment en considération les apports importants des déterminants sociaux de la santé a des implications dérangeantes en matière de politiques publiques. Cela exige de déplacer l'accent mis sur un réseau de santé axé sur la maladie vers une société axée sur le mieux-être fondé sur la prévention de la maladie et des maladies chroniques, en réglant diverses situations sociales et économiques (depuis la pauvreté jusqu'au logement) qui ont une influence sur la santé. Au Canada, bien que depuis trente ans on ait beaucoup parlé de déterminants sociaux de la santé dans les discours et publications entourant les politiques, ce changement de priorité ne s'est pas complètement réalisé.

Comme plusieurs déterminants de la santé se trouvent à l'extérieur du réseau de soins de santé, la stratégie pour améliorer la santé exige du travail de la part des intervenants sociaux dans le secteur public, privé et à but non lucratif, dans des domaines tels que le soutien du revenu, la justice, le logement, l'éducation, les transports, les services sociaux et communautaires (par exemple, les services de garde), qui, jadis, n'étaient pas perçus comme liés à la santé. Dans certains cas, négliger ces domaines pourrait exercer une influence négative sur l'état de santé des femmes par l'entremise de la violence qui leur est faite, comme on le voit ci-dessous (Figure 1). 
FIGURE 1 : L'EFFET MÉDIATEUR DE LA VIOLENCE

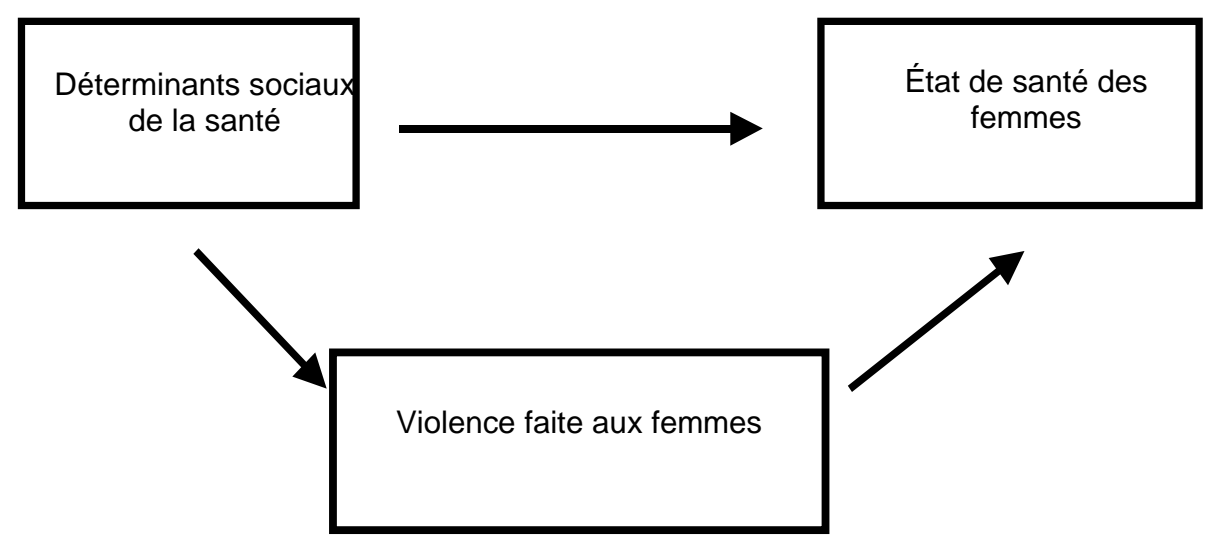

\section{VIOLENCE FAITE AUX FEMMES PAR UN CONJOINT}

Depuis plusieurs décennies déjà, les chercheurs tentent de saisir les causes profondes de la violence, en particulier de la violence conjugale. II s'agit évidemment d'un problème complexe et multidimensionnel, où aucun facteur ne peut prétendre être «la » seule explication (Gill, 2006). Une des nombreuses voies possibles pour mieux comprendre la question de la violence faite aux femmes pourrait donc être de l'aborder à la lumière d'une discussion axée sur les déterminants sociaux de la santé.

Comme on l'a indiqué plus haut, les liens entre divers déterminants sociaux et l'état de santé des individus (nous nous attardons ici aux femmes) et des collectivités ont été assez bien étayés, et on ne peut plus ignorer les données se rapportant à la flèche supérieure dans la figure 1 ci-dessus. Dernièrement, il a été admis ( $y$ compris par l'Organisation mondiale de la santé) que la violence conjugale nuit à la santé des femmes qui en sont victimes, étant donné que celles-ci en gardent souvent des séquelles physiques et psychologiques profondes. Nous savons que les femmes victimes de violence font effectivement plus appel aux services de santé et qu'elles sont plus sujettes au disfonctionnement social que les femmes qui n'ont pas subi de violence (Cohen et Maclean, 2003). Les femmes victimes de violence conjugale ont aussi plus de difficulté à dormir, souffrent plus de dépression ou de crises d'anxiété et ont tendance à consommer des somnifères, des calmants et des antidépresseurs. II reste donc peu d'analystes qui doutent de l'existence du lien entre la violence et une mauvaise santé, comme l'illustre la flèche de droite dans la figure 1 ci-dessus ${ }^{3}$.

3. Signalons que la violence conjugale peut aussi avoir des conséquences sérieuses chez les enfants qui en sont témoins. En comparant les réponses de ceux qui ont dit avoir grandi dans des foyers violents à celles des individus issus de foyers non violents, Berger (2002) a constaté que ceux qui provenaient des foyers abusifs étaient plus susceptibles de déclarer que leur santé n'était que mauvaise 
Ce qu'on a beaucoup moins étudié, ce sont les liens illustrés par la flèche de gauche dans la même figure : les liens entre les déterminants sociaux de la santé et la violence faite aux femmes. Bien que nous disposions de certaines données, nous devons, dans la poursuite de cette discussion, parler de certaines hypothèses ou d'opinions averties pour ce qui est de l'influence des facteurs tels que l'éducation, le revenu, le logement ou le niveau du soutien social sur la victimisation des femmes dans leurs relations conjugales. II nous faut toutefois faire preuve de prudence ici, car il importe de ne pas véhiculer l'image stéréotypée selon laquelle la violence ne touche que les femmes des classes les moins bien nanties, pauvres, vivant dans des foyers sans éducation ou dans des complexes résidentiels subventionnés. Nous savons qu'en réalité la violence à l'endroit des femmes survient dans toutes les classes sociales et qu'aucun groupe ethnique ou professionnel n'en est à l'abri. Toutefois, on constate au Canada que le taux de violence conjugale est plus élevé chez les ménages dont le revenu est inférieur à $30000 \$$ que chez ceux dont le revenu est de $60000 \$$ ou plus (Pottie Bunge, 2000). De même, Berger (2002) rapporte des taux de violence conjugale de $16 \%$ chez les ménages canadiens disposant d'un revenu de moins de 20000 \$, contre $4 \%$ chez les ménages disposant d'un revenu de 75000 \$ ou plus.

Nous savons aussi que les hommes peuvent être - et sont - victimes de violence perpétrée par leurs conjointes (Strauss, 1999), mais le fait que le taux de blessure dans ces situations (conséquences de violence conjugale) est considérablement plus élevé chez les femmes, justifie, à notre avis, que l'on met l'accent sur la violence faite aux femmes. Selon l'Enquête sociale générale (ESG) de Statistique Canada de 2004, les femmes victimes de violence conjugale sont au moins deux fois plus susceptibles de subir des blessures que les victimes masculines. Les femmes sont aussi trois fois plus susceptibles de craindre pour leur vie et deux fois plus sujettes aux agressions violentes répétées. Globalement, l'enquête révèle que les femmes sont plus susceptibles de subir des formes plus graves de violence conjugale que les hommes (qui déclarent rarement avoir fait appel à des soins médicaux à la suite de tels incidents). Les données relatives à l'une des formes les plus extrêmes de violence sont claires quant aux différences des conséquences selon le sexe : dans presque tous les cas canadiens de meurtre-suicide touchant des conjoints, la femme est la victime assassinée par le conjoint. Depuis 1991, on rapporte uniquement $2 \%$ de victimes masculines d'assassinat/suicide aux mains de leur conjointe. Globalement, il est évident que les femmes sont plus susceptibles de subir des formes plus graves de violence conjugale que les hommes (Tutty et Goard, 2002).

comparativement aux autres Canadiens et Canadiennes. Lors de son sondage téléphonique semiannuel, Berger a aussi trouvé des indices selon lesquels la violence au foyer pouvait se transmettre de génération en génération. 
TABLEAU 2

QUELQUES FAITS AU SUJET DE LA VIOLENCE CONJUGALE

- Les femmes subissent des formes de violence plus graves que les hommes.

- $\quad 16 \%$ des femmes qui ont été victimes de violence au cours des cinq dernières années ont été agressées sexuellement.

- $\quad 54 \%$ des femmes qui ont subi de la violence ont déclaré que celle-ci était survenue plus d'une fois.
- $\quad 13 \%$ des femmes qui ont subi de la violence ont fait appel à des soins médicaux.

- $8 \%$ des femmes blessées ont rapporté une fausse-couche.

- $\quad 97 \%$ des victimes de meurtre-suicide sont les conjointes.

- $\quad 44 \%$ des femmes qui ont subi la violence rapportent des blessures en découlant.

Source : Données de l'ESG rapportées par le Centre canadien de la statistique juridique (2005).

L'ESG de 2004 estime que $7 \%$ des femmes (et $6 \%$ des hommes) ont, dans une relation conjugale actuelle ou antérieure, fait face à de la violence conjugale au cours de la période de 2000 à $2004^{4}$. Au Canada, la violence conjugale est plus élevée chez les jeunes adultes (de 15 à 24 ans) dans les relations nouvelles ( 3 ans ou moins), et chez ceux qui vivent comme conjoints de fait. Les personnes qui vivent avec un gros buveur (quelqu'un qui consomme cinq verres ou plus à au moins cinq reprises au cours d'un mois) sont aussi six fois plus susceptibles de subir de la violence conjugale que celles qui vivent avec des buveurs raisonnables. Deux enquêtes sur l'agression révèlent aussi que la grossesse et les tentatives de quitter un partenaire violent augmentent le risque de violence conjugale (Ministres responsables de la condition féminine à l'échelle fédérale-provinciale et territoriale, 2002).

\section{LIENS POSSIBLES ENTRE LES DÉTERMINANTS SOCIAUX DE LA SANTÉ ET LA VIOLENCE CONJUGALE}

\section{EMPLOI ET REVENU}

Un facteur de risque qui semble, au niveau social, lié à la violence entre personnes est l'inégalité des revenus (Gartner, 1990). On peut extrapoler de cette observation que la violence entre les conjoints pourrait être plus susceptible de survenir dans une relation où il y a un écart plutôt important entre le revenu de chacun des partenaires, ou chez un couple où l'écart entre les capacités de s'approprier des ressources est très grand entre les partenaires. Ainsi, ne pas disposer de son propre revenu pourrait non seulement faire courir à la femme le risque de dépendance financière envers son partenaire, mais peut-être aussi le risque de devenir la victime de son conjoint. Bowlus et

4. Le sondage Berger Population Health Monitor (Sondage Berger, 2002) constate que $9 \%$ des Canadiens et Canadiennes de 19 ans et plus ont déclaré subir un certain niveau de violence de la part de leur époux ou partenaire. Chez les femmes, $13 \%$ ont déclaré subir de la violence d'un époux/partenaire contre $5 \%$ chez les hommes. 
Seitz (2005) ont constaté que les maris étaient effectivement plus susceptibles d'agresser des femmes sans emploi. II reste que les études canadiennes sur la violence conjugale ne se sont pas encore systématiquement penchées sur les liens possibles entre la violence dans le couple et l'écart entre les revenus et ressources des partenaires.

Chose intéressante, selon MacMillan et al. (1999), bien que la participation de la femme au marché du travail réduise le risque de violence quand son conjoint a aussi un emploi, cela accroît le risque quand son partenaire masculin n'a pas d'emploi. De même, d'après l'ESG, les taux de violence varient selon la principale activité du conjoint de la victime (Centre canadien de la statistique juridique, 2004). Celles dont le conjoint est à la recherche d'un emploi rémunéré sont plus susceptibles de subir de la violence conjugale que celles dont le partenaire a un emploi (10\% contre $4 \%$ ). Comme le note Johnson (1996), le chômage du conjoint peut parfois précipiter la violence conjugale. Enfin, l'ESG constate que non seulement l'accusé mais également la victime de violence conjugale, sont moins susceptibles d'avoir un emploi que les personnes mariées de la population en général (Centre canadien de la statistique juridique, 2004).

Il semble donc que les questions de l'emploi et du niveau de revenu ont un certain lien, probablement complexe, avec la violence conjugale. À tout le moins, on peut raisonnablement présumer que de faibles niveaux d'emploi et de revenu peuvent augmenter la tension et réduire les occasions de quitter une relation violente. Ce dernier point est évidemment particulièrement important pour comprendre pourquoi les femmes sont souvent réticentes à l'idée de quitter une relation violente. Comme elles ont en général un revenu moins élevé, les femmes sont souvent obligées, à cause des réalités financières qui permettent d'assurer la subsistance des enfants, de rester avec un conjoint violent ou de lui revenir, risquant ainsi d'être à nouveau victimes.

\section{RÉSEAU DE SOUTIEN SOCIAL}

Il est assez étonnant de constater qu'il y a peu de recherches sur la violence conjugale mettant en valeur les réseaux de soutien social. Récemment, en participant à un congrès sur la recherche en matière de violence familiale, soit la neuvième édition de la International Family Violence Research Conference, au New Hampshire ${ }^{5}$, nous avons constaté que la plupart des chercheurs présents n'avaient pas inclus le soutien social dans leur cadre analytique, plusieurs préférant s'en remettre surtout à une batterie d'outils psychologiques axés sur les individus et excluant des données mesurées relatives au milieu ${ }^{6}$.

Nous savons que, malgré le développement d'un certain nombre de services institutionnels, les victimes de violence conjugale se tournent surtout vers des appuis informels plutôt que formels. La plupart du temps, les victimes se confient à des proches, comme un membre de la famille, une amie ou une voisine (Centre canadien de la

5. Pour en savoir davantage au sujet de ce congrès, consulter : http://www.unh.edu/frl/conferences/2005/

6. À ce sujet, se reporter à W.S. Dekeseredy et M.D. Schwartz (2003). 
statistique juridique, 2005). Ne pas disposer d'un tel confident peut faire en sorte qu'il sera beaucoup plus difficile de prendre des mesures pour mettre fin à la violence. On peut donc considérer qu'un réseau de soutien faible et un relatif isolement social sont aussi des facteurs potentiels de risque pour la violence conjugale. Cependant, il reste de nombreuses dimensions à étudier à cet égard, par exemple, la capacité de raconter les violences subies à une personne de confiance.

\section{ÉDUCATION}

Les données fournies par l'ESG portent à croire, peut-être étonnamment, qu'il n'y a pas de lien entre le taux de violence conjugale et le niveau de scolarité de la victime ou de l'agresseur. Cependant, nous pouvons raisonnablement postuler qu'un niveau de scolarité plus élevé pourrait aider une femme victime de violence à repérer les services adéquats dans la communauté et dans le réseau de justice pénale, et à y accéder une fois la violence déclarée. Toutes proportions gardées, cela pourrait signifier qu'une femme instruite est peut-être moins susceptible de rester dans une relation violente (et donc moins susceptible d'être à nouveau victime du même partenaire).

D'autre part, une étude réalisée à l'université McMaster par l'économiste JoAnn Kingston-Riechers (1998) s'est penchée sur le lien entre le revenu et l'agression des conjointes. Elle a constaté qu'il n'y avait pas de forte corrélation entre le revenu et l'agression, mais que le lien entre le niveau de scolarité et l'agression était évident. En d'autres mots, l'agression serait plutôt liée à de faibles niveaux de scolarité qu'à la richesse ou à la pauvreté.

Du point de vue de l'agresseur, on pourrait croire qu'un homme plus instruit serait plus conscient des conséquences sociales possibles découlant d'une agression (ultimement, la prison) et qu'il aurait plus à perdre en empruntant cette voie. Par contre, bien que cette hypothèse puisse sembler rationnelle, elle demeure une idée à explorer à l'avenir et, à notre connaissance, il manque à l'heure actuelle des données empiriques pour la soutenir.

\section{MODE DE VIE ET PRATIQUES D'HYGIÈNE}

On sait que l'abus d'alcool et de drogue est associé à l'incidence de la violence conjugale. Le rôle de l'alcool (surtout de sa consommation excessive) a été bien documenté (Sumner et Parker, 1995). Quoique l'alcool ne soit pas la cause de la violence entre les conjoints, sa consommation interagit avec d'autres facteurs pour augmenter le risque de violence conjugale (Gelles et Straus, 1988; Gelles et Cavanaugh, 2005). Dans l'Enquête sur la violence envers les femmes de 1993, on rapporte que 29 \% des femmes qui avaient subi une agression violente ont indiqué que la violence commençait habituellement après que leur partenaire eut consommé de l'alcool. L'ESG de 2004 indiquait de même que, dans le cas de $35 \%$ des incidents de violence conjugale, la personne responsable de l'agression avait consommé de l'alcool (la proportion est de $44 \%$ dans les cas où la victime était une femme). 
On dispose de renseignements moins fiables au sujet du lien entre la consommation de drogues illicites et la violence conjugale, mais il est vraisemblable que les tendances soient similaires à celles se rapportant à l'alcool. Pour une femme, avoir une relation intime avec un utilisateur de drogues illicites ou en consommer elle-même, pourrait la placer dans différentes situations où la violence risque de survenir dans le couple.

Pour ce qui est de certaines pratiques d'hygiène personnelle positives, par exemple l'activité physique régulière et une bonne hygiène de vie, on ne connaît aucune recherche qui ait tenté d'établir systématiquement le lien entre ces pratiques et la violence conjugale. Mais, encore une fois, nous pouvons à tout le moins postuler que ces pratiques pourraient véhiculer une certaine maîtrise sur sa vie et que cette impression pourrait être utile lorsqu'il s'agit de trouver des moyens efficaces de mettre fin à la violence $^{7}$.

\section{CULTURE/ETHNICITÉ}

Dans le contexte canadien, l'ESG indique que les personnes autochtones sont trois fois plus susceptibles d'être victimes de violence conjugale (21\% contre $7 \%$ chez les non-autochtones). La violence conjugale est aussi plus grave chez les femmes autochtones, car elles sont plus susceptibles d'être battues, étranglées ou menacées à la pointe d'un fusil ou d'un couteau. Les taux d'agression sexuelle, de blessure, et le nombre de femmes qui craignent pour leur vie sont aussi plus élevés chez les victimes autochtones.

Nous ne croyons pas que ces données indiquent que la violence conjugale est inhérente à la culture autochtone ou qu'elle y est plus tolérée. Nous considérons plutôt ces chiffres comme des indicateurs symptomatiques de l'état d'oppression et de marginalisation que subissent au Canada les autochtones en général, et les femmes autochtones en particulier. Nous savons que les autochtones canadiens ont tendance à disposer de niveaux moins élevés de revenu, d'emploi et d'éducation, et à connaître des taux d'abus d'alcool plus élevés. La grande prévalence de la violence à l'endroit des femmes dans leurs communautés pourrait donc constituer une des meilleures indications qu'il nous faut étudier plus à fond les liens entre les déterminants sociaux de la santé et la violence faite aux femmes par leurs conjoints violents.

\section{CONCLUSION}

L'objectif de cet article est de stimuler une réflexion au sujet des déterminants sociaux de la santé et de leur possible influence sur la violence conjugale (à l'endroit des femmes) en tant que forme commune d'agression. Il est désormais évident que les déterminants sociaux de la santé exercent une influence considérable sur l'état de santé des personnes. II est aussi assez bien établi qu'être victime de violence conjugale peut

7. Un des lecteurs de ce document a relevé la possibilité que l'exercice physique constructif pourrait désamorcer la violence chez les agresseurs. Ce point de vue intéressant aurait besoin d'être étudié de plus près! 
causer chez les femmes des conséquences négatives, dont les séquelles peuvent durer longtemps. II y a toujours un flagrant besoin de recherche approfondie au sujet du lien entre plusieurs déterminants sociaux de la santé et l'agression des femmes par leur partenaire intime. II est vraisemblable que plusieurs de ces liens sont complexes et ne sont pas simplement linéaires ou unidirectionnels.

Si nous pensons aux logements inadéquats et non sécuritaires par exemple, nous pouvons raisonnablement postuler que ces logements qui ne répondent pas aux normes, peuvent à la fois être une conséquence et un facteur menant à la violence conjugale, comme on le voit à la figure 2 ci-dessous.

FIgURE 2 : LE LOGEMENT ET LA VIOLENCE FAITE AUX FEMMES

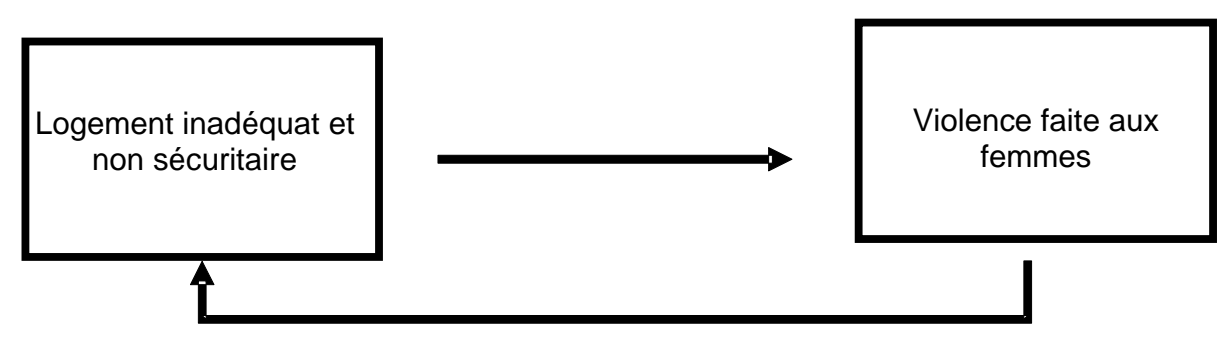

La violence conjugale peut évidemment contribuer à des conditions de logement inadéquates, voire à l'itinérance chez les femmes (Neal, 2004). On peut aussi croire que certaines conditions de logement (par exemple plusieurs familles sous un même toit, ce qui n'est pas rare chez les autochtones confrontés aux questions de logement) peuvent engendrer des tensions qui mèneraient à des incidents de violence conjugale. Du point de vue des politiques sociales, cela implique qu'en travaillant à rendre le logement plus abordable pour les Canadiens et Canadiennes à faible revenu, on pourrait contribuer à prévenir la violence conjugale de plusieurs façons, y compris en permettant aux femmes, du point de vue financier, d'accéder plus facilement à des options de logement adéquates lorsqu'elles quittent une relation violente.

De la même manière, la création au Canada d'un réseau universel de centres pour la petite enfance pourrait aussi constituer une initiative en matière de politiques sociales qui contribuerait indirectement à réduire les nouveaux incidents de violence envers les femmes, en leur permettant de concilier plus facilement le travail et le soin des enfants après avoir quitté un partenaire violent. Des mesures de développement communautaire pourraient aider à renforcer les réseaux sociaux locaux et à réduire l'isolement que connaissent certaines femmes qui courent un risque de violence - ou la subissent déjà. D'autres mesures en matière de politiques sociales destinées à répondre aux besoins des femmes en matière de revenu, et, plus généralement, à réduire les 
inégalités des revenus dans la société, aideront vraisemblablement (à la lumière de ce que nous savons maintenant) non seulement à améliorer l'état de santé de la population en général (Vaillancourt et al., 2004), mais pourraient aussi aider à prévenir et à réduire différentes formes de violence, y compris celle que subissent les femmes aux mains de leurs conjoints.

Comme on le voit, il nous importe de reconnaître la complexité des questions qui entourent la violence faite aux femmes. En prenant conscience des liens possibles entre les déterminants sociaux de la santé et la violence faite aux femmes par leurs conjoints, on est plus en mesure de proposer et d'appuyer des recherches et des initiatives en matière de politiques sociales qui pourraient aider à réduire l'incidence de la violence conjugale, et à créer un pays globalement plus sûr et en meilleure santé.

\section{RÉFÉRENCES BIBLIOGRAPHIE}

Berger, E. (2002). National Report on Abuse: Summary of Findings from the Berger Population Health Monitor, Ottawa, Santé Canada.

Bowlus, A.J., et S. SEITZ (2005). Domestic Violence, Employment and Divorce.

[http://qed.econ.queensu.ca/pub/faculty/seitz/abuse11.pdf].

Centre Canadien de la statistique JuRidique (2004). Brzozowski, J. (dir.), La violence familiale au Canada : un profil statistique 2004, Ottawa, Statistique Canada.

Centre CANADIEN DE LA STATISTIQUe JURIDIQUe (2005). Aucoin, K. (dir.). La violence familiale au Canada : un profil statistique 2005, Ottawa, Statistique Canada.

Cohen, M., et H. MACLEAN (2003). « Violence against Canadian Women », dans M. DesMeULeS et D. SteWART (dir.), Women's Health: Surveillance Report, Ottawa, Institut canadien d'information sur la santé, p. 45-46.

COMITÉ CONSULTATIF FÉDÉRAL-PROVINCIAL-TERRITORIAL SUR LA SANTÉ DE LA POPULATION (1999). Pour un avenir en santé: Deuxième rapport sur la santé de la population canadienne, Ottawa, Ministère des Travaux publics.

DeKESEREDY, W.S., et M.D. SchwARTZ (2005). « Backlash and Whiplash: A Critique of Statistics Canada's 1999 General Social Survey on Victimization », Online Journal of Justice Studies, vol. 1, $\mathrm{n}^{0}$ 1. [http://ojjs.icaap.org/issues/1.1/dekeseredy-schwartz.html].

GARTNER, R. (1990). «The Victims of Homicides: A Temporal and Cross-national Comparison », American Sociological Review, $n^{\circ} 55$, p. 92-106.

Gelles, R., et M. Cavanaugh (2005). « Association is not Causation: Alcohol and other Drugs do not Cause Violence », dans D. Loseke, R. Gelles et M. Cavanaugh (dir.), Current controversies on family violence, Thousand Oaks, Sage, p. 175-190.

Gelles, R., et M. Strauss (1988). Intimate Violence: The Causes and Consequences of Abuse in the American Family, New York, Simon and Schuster. 
GILL, C. (2006). «Violence between Intimate Partners: Understanding Theories and their Links to Intervention Strategies », dans M. HAMPTON et N. GERRAD (dir.), Intimate partner violence: reflections on experience, theory and policy, Toronto, Cormorant Books, p. 47-66.

Greaves, L., O. HANKIVSKY, et J. Kingston-RiEChERS (1995). Selected Estimates of the Cost of Violence against Women, London (Ontario), Centre for Research on Violence Against Women and Children.

Johnson, H. (1996). Dangerous Domains: Violence against Women in Canada, Toronto, Nelson.

KINGSTON-RIECHERS, J. (1998). The Association between Socio-Economic Characteristics and the Frequency of Wife Assault, Working Paper Series, département d'économie, McMaster University.

[http://socserv2.socsci.mcmaster.ca/ econ/rsrch/papers/archive/deptwp9806.pdf].

LALONDE, M. (1974). Nouvelle perspective de la santé des Canadiens, Ottawa, Santé et Bienêtre Canada.

MacMillan, R., et R. GARTNER (1999). « When She Brings Home the Bacon: Labor-force Participation and the Risk of Spousal Violence against Women », Journal of Marriage and the Family, vol. $61, \mathrm{n}^{\circ} 4$, novembre.

MINISTRES RESPONSABLES DE LA CONDITION FÉMININE À L'ÉCHELLE FÉDÉRALE-PROVINCIALE ET TERRITORIALE (2002). Évaluation de la violence contre les femmes: un profil statistique, Ottawa, Condition féminine Canada.

NeAL, R. (2004). Voices: Women, Poverty and Homelessness in Canada, Ottawa, Organisation nationale anti-pauvreté.

Pottie Bunge, V. (2000). «Spousal Violence » dans V. Pottie Bunge et D. Locke (dir.), La violence familiale au Canada : un profil statistique 2000, Ottawa, Statistique Canada, p. 1119.

SANDEL, M. (2003). « 10 Things Every Social Worker Needs to Know About Domestic Violence », The New Social Worker, vol. 10, $\mathrm{n}^{\circ} 3$, été.

[http://www.socialworker.com/domesticviolence.htm]

Santé Canada, Agence de santé publique du CANAda (2005). Santé de la population. [http://www.phac-aspc.gc.ca/ph-sp/ddsp/determinants/].

StRAUSS, M.A. (1999). «The Controversy over Domestic Violence by Women: A Methodological, Theoretical, and Sociology of Science Analysis », dans X. ARRIAGA et S. OSKAMP (dir.), Violence in Intimate Relationships, Thousand Oaks (Californie), Sage, p. 17-44.

Summer, M., et H. PARKer (1995). Low in Alcohol: A Review of International Research into Alcohol's Role in Crime Causation, Londres, The Portman Group.

TUTTY, L., et C. GOARD (dir.) (2002). Reclaiming Self: Issues and Resources for Women Abused by Intimate Partners, Halifax, Fernwood/REVOLVE.

Vaillancourt, Y., F. Aubry, M. KEARNEY, L. ThÉRIAUlt, et L. Tremblay (2004). « Contribution of the Social Economy Toward Healthy Social Policy Reforms in Canada: A Quebec Viewpoint », dans D. RAPHAEL (dir.), Social Determinants of Health: Canadian Perspectives, Toronto, CSPI, p. 311-330. 
Wuest, J., et M. MERRITT-Gray (2004). « Family Violence as a Social Determinant of Health », dans J. Ruggerl et W. Yu (2004). Determinants of Health: An Atlantic Perspective, Fredericton, University of New Brunswick, p. 86-108. 